\title{
Ozonolysis of p-Benzoquinone II
}

\author{
ERLING BERNATEK and KNUT ASBJøRN STRAUMSGARD
}

\author{
Universitetets Kjemiske Institutt, Blindern-Oslo, Norway
}

\begin{abstract}
The ozonolysis of $p$-benzoquinone in chloroform at $3-4^{\circ}$ has been studied. By decomposition of the ozonized solution with water the following compounds were formed: Formic acid, glyoxylic acid, oxalic acid, maleic acid, mesoxalic dialdehyde, formaldehyde and carbon dioxide. A quantitative study of these compounds has been undertaken, and possible mechanisms for their formation are presented.
\end{abstract}

$\mathrm{I}^{\mathrm{n}}$

a preliminary communication ${ }^{1}$ (Part I of this series) one of the present authors discussed some aspects of the ozonolysis of $p$-benzoquinone in chloroform. The main result was the tentative establist ment of the unsaturated aldehyde $\mathrm{CHOCOCH}=\mathrm{CHCOCHO}$ as a decomposition product. An earlier investigation by Fichter, Jetzer and Leepin ${ }^{2}$ had led to the identification of formic acid among the products of this ozonolysis.

It soon became clear that the ozonolysis of $p$-benzoquinone was a rather complex affair and that the reaction products might be numerous. We have therefore performed a more detailed investigation of the products which occurred when the ozonolysis was carried out under certain specified conditions. It is appropriate to remark at this point that the qualitative and quantitative comrosition of the reaction mixture seems to be highily susceptible to even minor alterations in reaction conditions. We have thus in the present investigation not isolated any unsaturated aldehyde although the conditions of ozonolysis were not very different from those of the earlier investigation.

During the ozonization an ozonide separated as described before ${ }^{1,2}$, but no attempts were made to characterize this unstable and highly hydrolysable substance. If kept in air at room temperature for instance, it soon became a dark syrup in which after some time long needles of oxalic acid appeared.

The ozonized mixture was decomposed by shaking with cold water when the ozonide quickly went into solution and the active oxygen disappeared. The aqueous extract had a strong acidic reaction but contained only traces of hydrochloric acid (which have resulted from decomposition of the chloroform). In order to identify and determine the amount of volatile acids the aqueous solution was distilled at room temperature by passing nitrogen through it at low pressure and collecting the distillate in aqueous sodium hydroxide. 
Only formic acid could be found. A neutral substance in the decomposition solution was isolated by removing the acidic products in a column of a weakly basic ion-exchange resin. In the neutral eluate there was a substance which instantly reduced Tollens' reagent and which through its $p$-nitrophenylhydrazone was identified as mesoxalic dialdehyde. As it was found that the reaction mixture also contained non-volatile acid substances, the ion-exchange column after removing quantitatively the mesoxalic dialdehyde with water, was treated with $5 \%$ aqueous formic acid. The eluate contained a carbonyl compound which reacted with 2,4-dinitrophenylhydrazine to give a derivative in the form of yellow needles. The melting point of this product ( $190^{\circ}$ decomp.) was not changed after recrystallization so it may be concluded that only one carbonyl compound had been eluted with the dilute formic acid, viz., glyoxylic acid. Elution of the ion-exchange column with $2 \mathrm{~N}$ hydrochloric acid afforded finally oxalic and maleic acids. These two acids were, however, more easily isolated directly from the decomposition solution.

Beside the two aldehydes, mesoxalic dialdehyde and glyoxylic acid, a third aldehyde was found but only in very small amounts. The derivative with dimedone and the colour with chromotropic acid proved that this third aldehyde was formaldehyde. It could be shown that this compound did not result from decomposition of the solvent or of impurities.

During the decomposition of the ozonized solution with water an evolution of carbon dioxide could be noticed. The aqueous extract gave only a very faint reaction on hydrogen peroxide.

In order to get a better picture of the fate of benzoquinone during ozonolysis and the mode of formation of the fragments, it was necessary to undertake a quantitative determination of the products. Some of the analytical methods available were not very accurate but it is felt that a fairly good estimate of the relative amounts in which the above-mentioned degradation products occurred has been secured.

Formic acid was determined as mentioned before by distillation into aqueous sodium hydroxide at low temperature and pressure.

Mesoxalic dialdehyde was determined by evaporation of the eluate from the ionexchange column and weighing the residue. This is clearly a minimum value as there always is a certain loss of substance in the column. Furthermore the aldehyde is somewhat volatile with water vapour and in addition to this only that part of the eluate which contained appreciable amounts of the aldehyde was evaporated. To concentrate the total volume of the neutral eluate would have been almost a practical impossibility.

Glyoxylic acid could be precipitated as the dinitrophenylhydrazone but the precipitation was far from quantitative. To avoid this source of error and to avoid the loss in the column glyoxalic acid was determined by difference. The decomposition solution was in this case evaporated directly in a vacuum desiccator over potassium hydroxide to constant weight. From this value were subtracted the amounts of the non-volatile products mesoxalic dialdehyde, oxalic acid and maleic acid. The amount found in this way must be a maximum value since contaminations and eventual minor amounts of unidentified substances are included and - which is important - because the mesoxalic dialdehyde is represented by a minimum value.

Oxalic acid was precipitated in accordance with the usual analytical method as the calcium salt.

Maleic acid was determined in the filtrate from the oxalate determination by precipitation as the barium salt. This salt has the drawback to be somewhat soluble in water so the filtrate had to be measured and the amount of dissolved barium maleate calculated. An alternative method of analysis was to oxidize the concentrated decomposition solution

Acta Chem. Scand. 13 (1959) No. 1 
with hydrogen peroxide. Maleic acid is much slower oxidized by this reagent than the other compounds present. The residue after evaporating the oxidation mixture consists of pure maleic acid. Some loss is inevitable in this last procedure but the two methods give results of the same order of magnitude.

Several methods were tried for the determination of carbon dioxide. Absorption in barium hydroxide followed by a rapid filtration of the precipitated barium carbonate was chosen.

In Table 1 the analytical results are given in relation to one mole of benzoquinone and in addition the percentages of recovered carbon are calculated.

\section{Product}

Formic acid

Mesoxalic dialdehyde

Glyoxylic acid

Oxalic acid

Maleic acid

Carbon dioxide
Table 1.

Mole/mole
benzoquinone

1.65
0.13
1.16
0.03
0.06
0.16
Recovered carbon, \%

39.0

1.4

4.5

2.7

It will be seen that account can be made for about $83 \%$ of the carbon in the starting material. The deficiency of about $17 \%$ may in part be explained by imperfection of our analytical methods, but it is also highly probable that some of the benzoquinone is oxidized directly to water and carbon dioxide during the ozonization. As ozone is not absorbed quantitatively by benzoquinone some of it escapes with the effluent oxygen and interferes with attempts to determine directly formed carbon dioxide. In addition to this comes a small amount of material still contained in the chloroform layer after extraction with water. Per gram of benzoquinone this amounts to about $20 \mathrm{mg}$.

The maleic acid can only be derived from a monozonide of benzoquinone. In the diozonide of course both carbon-carbon double bonds are broken and no maleic acid can result. During ozonolysis there was always passed through the solution ozone in excess of that calculated for reaction with both double bonds but nevertheless some monozonide must have existed. This is evidently due to the already mentioned incomplete absorbtion of ozone by benzoquinone and to the fact that some ozone is decomposed by the solvent before it can react with the quinone.

The monozonide of benzoquinone may be a normal ozonide like I.

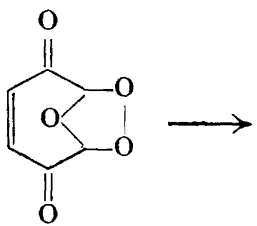

I<smiles>O=COC(=O)/C=C\C(=O)C=O</smiles>

II<smiles>CC(O)/C=C\C(=O)C(=O)C=O</smiles>

III<smiles>O=C(O)/C=C\C(=O)O</smiles>

IV 
It is well known that normal ozonides like other peroxy compounds may undergo rearrangements, which in our case would lead from I to II. Another possibility is that benzoquinone via anomalous ozonolysis more directly is converted to II. The latter structure will be recognized as a mixed anhydride between formic acid and the unsaturated ketoaldehydic acid III. By treatment with water these two acids will be set free. Transformation of III into maleic acid (IV) obviously involves an oxidation. This may be effected by ozonide still present or, since maleic acid is formed only in minor amounts, by those small quantities of hydrogen peroxide present in the decomposition solution or maybe already during the ozonization. The aldehyde group is most probably oxidized to formic acid, less likely it seems to carbon dioxide and water. This last statement is in part based upon the fact that Dallwigk and Briner ${ }^{3}$ who investigated the ozonolysis of cinnamaldehyde found that the glyoxylic acid which was formed by treatment of the ozonide with water was further oxidized to formic acid and carbon dioxide by undecomposed ozonide.

When discussing the formation of the other products several reaction paths must be taken into consideration, the most conspicious of which are the two already mentioned, viz., formation of the normal ozonide with subsequent rearrangement and anomalous ozonolysis. Further may be mentioned direct hydrolytic cleavage of the ozonide and the so-called "acid-rearrangement" which is well known to occur among aldozonides and which may be represented as follows:

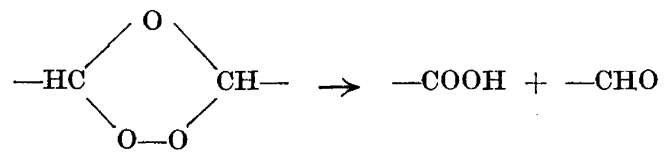

Still more numerous are of course the combinations of these reactions which may be possible with a diozonide. To postulate such combinations will, however, be rather speculative and therefore left out of serious consideration.

The main products, formic acid and glyoxylic acid, which are formed in rather overwhelming molar quantities as compared with the other products are most probably formed by symmetrical * double rearrangement of the normal diozonide or by symmetrical anomalous ozonolysis:

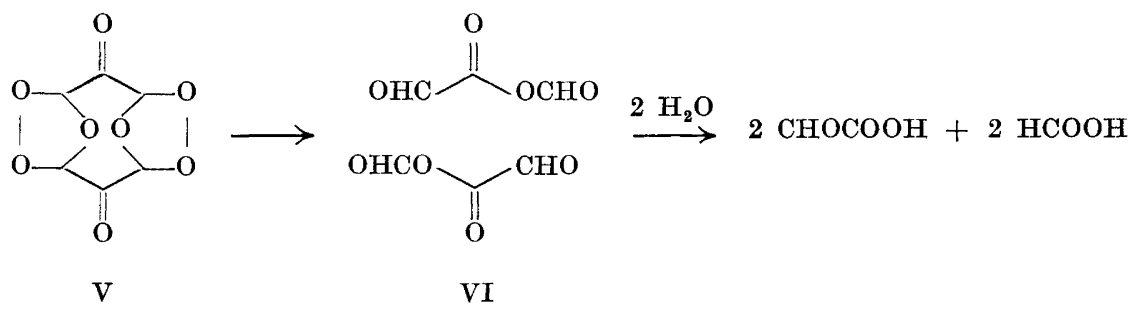

* Symmetrical in the sense that the two migrating oxygen atoms become adjacent to different carbonyl groups. In the same sense unsymmetrical rearrangement means that the two oxygens become adjacent to the same carbonyl group.

Acta Chem. Scand. 13 (1959) No. 1 
It is difficult to discern between the two mentioned possibilities, but it may be concluded that at least a part of the reaction goes via the normal diozonide as the peroxidic substance which separated during ozonization could be kept for some time at $0^{\circ}$ without decomposition. At room temperature, however, it dissolved in the chloroform after a short time. Simultaneously carbon monoxide began to evolve which can be taken as an indication of the actual existence of structures like VI. Mixed anhydrides of formic acid are known to decompose with the evolution of carbon monoxide under anhydrous conditions. If 0.58 mole of benzoquinone undergoes the above degradation there will be produced the 1.16 moles of glyoxylic acid actually found and 1.16 moles of formic acid.

Carbon dioxide and mesoxalic dialdehyde are formed in roughly equimolecular amounts (especially if we take into account that the found amount of mesoxalic dialdehyde is a minimum value) and very likely through an unsymmetrical double rearrangement of the normal ozonide or by unsymmetrical anomalous ozonolysis:

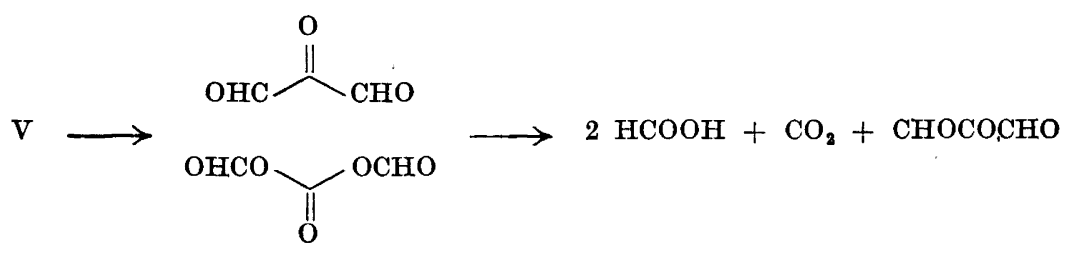

Assuming that 0.16 moles of benzoquinone reacts in this way the products will be 0.16 moles of carbon dioxide, 0.16 moles of mesoxalic dialdehyde and 0.32 moles of formic acid.

Oxalic acid contains carbon in a higher oxidation state than the other products (except carbon dioxide) and its formation is difficult to explain without further oxidation of the decomposition products. A possible mechanism is the symmetrical double acid-rearrangement of the normal diozonide:<smiles>CCCCCCCCCCC(=O)OC(=O)C(=O)O</smiles>

Oxidation of the primarily formed mesoxalic monoaldehyde probably takes place during the ozonization. If the rearrangement starts at an early stage of the reaction, ozone and small amounts of water formed by complete breakdown of benzoquinone may easily convert the ketoaldehydic acid to oxalic acid and formic acid. 0.015 moles of benzoquinone reacting as described give 0.03 moles of oxalic acid and 0.03 moles of formic acid.

When adding together the mentioned molar amounts of formic acid and remembering that 0.06 moles of maleic acid are accompanied by 0.12 moles 
of formic acid we get the total of 1.63 moles of formic acid. This figure corresponds very well with the found amount of 1.65 moles and therefore lends support to the soundness of the presented paths of reaction. Addition of the consumed molar amounts of benzoquinone gives 0.82 moles. (Compare ca. $83 \%$ recovered carbon).

The problem of formaldehyde formation has not been discussed yet, mainly because the amount of this aldehyde is so small that it does not affect the overall picture of degradation. If we regard the molecular formulae for benzoquinone and formaldehyde $\mathrm{C}_{6} \mathrm{H}_{4} \mathrm{O}_{2}$ and $\mathrm{CH}_{2} \mathrm{O}$, it will be noticed that carbon in both compounds has the formal oxidation stage zero. That means that the particular carbon atom in the benzoquinone skeleton going to form formaldehyde must lee severed retaining the mean formal oxidation stage. Obviously this can only be attained by hydrolytic cleavage of at least one carbon-carbon bond, for instance in the following way:

$$
\mathrm{HOH}+-\mathrm{CO}-\mathrm{CHO} \longrightarrow-\mathrm{COOH}+\mathrm{HCHO}
$$

The fragment to be hydrolysed must be a rearrangement product from one of the ozonides.

\section{EXPERIMENTAL}

Ozonization procedure. The source of ozone was an apparatus built in this institute and consisted of ten Berthelot tubes; voltage $11000 \mathrm{~V}$. Through the apparatus was led a stream of dry tank oxygen at the rate of $30 \mathrm{l} / \mathrm{h}$. At this rate of flow a yield close to $2 \mathrm{~g}$ $\mathrm{O}_{3} / \mathrm{h}$ was obtained which corresponds to an ozone concentration of ca. $4.8 \%$ (we1ght).

A good commercial grade of chloroform was used as a solvent. This was dried with calcium chloride and distilled with rejection of rather large head and tail fractions. The $p$-benzoquinone was a recrystallized Eastman-Kodak product.

As reaction vessel a gas washing bottle with fritted bottom for the distribution of gas was employed. During ozonization the bottle was placed in an ice-bath. The ozonization mixture (usually $5 \mathrm{~g}$ of quinone in $150 \mathrm{ml}$ of chloroform or $1 \mathrm{~g}$ of quinone in $50 \mathrm{ml}$ of chloroform) would then during the reaction attain a temperature of 3 to $4^{\circ}$. The end point of the reaction could not be determined by the sudden appearance of unreacted ozone (as ozone was passing through the solution already from the outset) but could be judged with fair accuracy from the disappearance of the yellow quinone colour. At this point an ozonide had separated at the walls of the vessel as a white sticky mass, the solution being light greenish. If this mixture was kept at room temperature the separated ozonide went into solution. Strips of filter paper moistened with a palladium chloride solution, which were suspended in the reaction vessel above the liquid, blackened in a short time.

The reaction mixture was decomposed by shaking with ice-cold water and the chloroform layer was extracted a few more times with additional amounts of water. The ozonide went quickly into solution and the chloroform thereafter gave no reaction on active oxygen. On the other hand the aqueous solution (hereafter referred to as "decomposition solution") gave a faint reaction on hydrogen peroxide with titanium reagent. If the extracted chloroform was dried with sodium sulphate and evaporated to dryness there remained a solid brownish residue amounting to about $20 \mathrm{mg} / \mathrm{g}$ benzoquinone. The nature of this residue has so far not been investigated.

Formic acid. Some of the decomposition solution was distilled and the distillate exactly neutralized with aqueous sodium hydroxide. To this was added a hot concentrated solution of S-benzylthiouronium chloride. The mixture was immediately cooled and inoculated with a small crystal of S-benzylthiouronium formate. In a few hours white crystals had separated which had m. p. $146^{\circ}$. Mixed m. p. with an authentic sample showed no depression.

For the quantitative determination aliquots of the decomposition solutions from ozonization of $1.000 \mathrm{~g}$ portions of benzoquinone were distilled. The distillations were in this

Acta Chem. Scand. 13 (1959) No. 1 
case performed at low pressure and room temperature while a slow stream of nitrogen was passed through the solution. Formic acid was absorbed directly in a $0.1000 \mathrm{~N}$ solution of sodium hydroxide and the resulting mixture back-titrated with hydrochloric acid. As a mean of the determinations it was found that $1.000 \mathrm{~g}$ of benzoquinone produced 15.2 mmoles of formic acid.

Mesoxalic dialdehyde. The decomposition solution from $5.00 \mathrm{~g}$ of benzoquinone was added to the same volume of ethanol and the mixture passed through a column of $\mathrm{De}_{\theta}$. Acidite $\mathrm{G}(3 \times 30 \mathrm{~cm})$. Before use the ion exchange resin had been treated with $1 \mathrm{~N}$ sodium hydroxide and washed neutral, first with tap water and finally with distilled water. The ethanolic decomposition solution was left in the column for about one hour and thereafter eluted with $50 \%$ ethanol. About $500 \mathrm{ml}$ of a neutral eluate which showed a strong reaction with Tollens' reagent was collected. A faint but very persistent tailing was characteristic of the elution and the volume of eluate actually containing traces of a neutral carbonyl compound was very large. The $500 \mathrm{ml}$ of eluate was evaporated to dryness in vacuo and formed a dry brittle mass of only faint colour. It could not be brought to crystallization and was difficult to purify in this state. The mean yield of the product was $500 \mathrm{mg}$. In order to prepare the $p$-nitrophenylhydrazone, an aqueous solution of the aldehyde was added to a hot solution of $p$-nitrophenylhydrazine in glacial acetic acid and the mixture heated on a water-bath for about one hour when a dark red substance separated. This substance was digested three times with glacial acetic acid and the undissolved part recrystallized from nitrobenzene. The dark brick-red derivative had no melting point but decomposed at about $297^{\circ}$; the decomposition point was, however, rather dependent upon the rate of temperature increase. (Found: C 52.0; $\mathrm{H} \mathrm{3.8;}$ $\mathrm{N}$ 24.4. Calc. for $\mathrm{C}_{21} \mathrm{H}_{17} \mathrm{~N}_{9} \mathrm{O}_{6}$ : $\mathrm{C} 51.3 ; \mathrm{H} \mathrm{3.5} ; \mathrm{N}$ 25.7.) The analytical values were rather high in carbon and low in nitrogen content, a fact which is attributed to the tenacious retaining of nitrobenzene by the tris-p-nitrophenylhydrazone. An authentic sample of mesoxaldialdehyde was prepared by ozonization of phorone and a derivative with $p$ nitrophenylhydrazine was made as described above. Also here a decomposition point of about $297^{\circ}$ was found and the analytical figures were close to those already mentioned. (Found: C 52.0; H 3.7; N 24.1.) The infrared spectra of the two derivatives proved that they were identical.

From the first acetic acid extract of our original $p$-nitrophenylhydrazone mixture a dark red substance separated. Decomposition occurred in the range 260 to $270^{\circ}$. (Found: $\mathrm{C} \quad 50.0 ; \mathrm{H}$ 3.8; $\mathrm{N}$ 21.8. Calc. for $\mathrm{C}_{9} \mathrm{H}_{7} \mathrm{~N}_{3} \mathrm{O}_{4}$ (mono-p-nitrophenylhydrazone): $\mathrm{C} \quad 48.9$; $\mathrm{H}$ 3.2; $\mathrm{N} 19.0$. Calc. for $\mathrm{C}_{15} \mathrm{H}_{12} \mathrm{~N}_{6} \mathrm{O}_{5}$ (bis-p-nitrophenylhydrazone): $\mathrm{C} 50.6 ; \mathrm{H} 3.4 ; \mathrm{N} \mathrm{23.6}$ ).

Glyoxylic acid. After removing the mesoxalic dialdehyde completely the ion-exchange column was treated with $5 \%$ aqueous formic acid. In the eluate a carbonyl compound appeared which reacted with 2,4-dinitrophenylhydrazine to give yellow needles; $\mathrm{m}$. $\mathrm{p}$. $190^{\circ}$ decomp. Recrystallized twice from water the melting point was unchanged. Mixed melting point with an authentic sample of glyoxylic acid 2,4-dinitrophenylhydrazone was

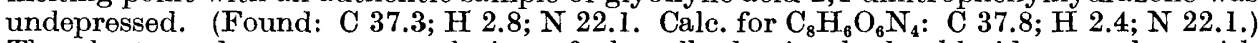
The eluate and an aqueous solution of phenylhydrazine hydrochloride gave brownish orange needles. Recrystallized from water (charcoal) they became light yellow; m. p. $137-138^{\circ}$ decomp. (Found: C 58.5; $\mathrm{H} \mathrm{4.9;} \mathrm{N} \mathrm{16.6.} \mathrm{Calc.} \mathrm{for} \mathrm{C}_{8} \mathrm{H}_{8} \mathrm{~N}_{2} \mathrm{O}_{2}$ : C 58.5; $\mathrm{H} \mathrm{4.9;}$ N 17.1.)

The decomposition solution from $1.000 \mathrm{~g}$ of benzoquinone was evaporated in a vacuum desiccator over potassium hydroxide to constant weight. Weight of the residue $1.197 \mathrm{~g}$. From this value was deducted the sum of the found amounts of mesoxalic dialdehyde, oxalic acid and maleic acid, in all $202 \mathrm{mg}$, which leaves us $995 \mathrm{mg}$ of glyoxylic acid monohydrate, i.e. $800 \mathrm{mg}$ of the anhydrous acid.

Oxalic acid. The decomposition solution from $1.000 \mathrm{~g}$ of benzoquinone was added to a solution of $2 \mathrm{~g}$ calcium chloride in $50 \mathrm{ml}$ of water and dilute aqueous ammonia added until $\mathrm{pH}$ reached $c a$. 6 . The crystalline precipitate was collected after one hour and dried at $110^{\circ}: 52 \mathrm{mg}$ of calcium oxalate monohydrate which corresponds to $32 \mathrm{mg}$ of oxalic acid. A larger amount of calcium oxalate prepared in this way was dissolved in $2 \mathrm{~N}$ hydrochloric acid and extracted with ether. The dried ethereal extract was evaporated when white crystals remained. These had m. p. $101^{\circ}$, undepressed upon admixture of an authetic sample of oxalic acid.

Maleic acid. The filtrate from the oxalate precipitation was concentrated to a small volume and a hot solution of $0.5 \mathrm{~g}$ of barium chloride in a few $\mathrm{ml}$ of water added when a 
white precipitate was formed. The mixture was cooled to about $15^{\circ}$ and filtered rapidly by suction. In a typical run the dried barium maleate weighed $44 \mathrm{mg}$ and the filtrate from this salt measured $20 \mathrm{ml}$. Now it is found in the literature ${ }^{4}$ that 100 parts of water at $14.5^{\circ}$ dissolves 0.584 parts of barium maleate monohydrate. The filtrate contains therefore $108 \mathrm{mg}$ of the anhydrous salt and the total of $153 \mathrm{mg}$ of barium maleate corresponds to $72 \mathrm{mg}$ of maleic acid. From the barium salt maleic acid could be set free and isolated. It was identified by the melting point which was undepressed upon admixture of an authentic sample.

Maleic acid could also be determined more directly. The decomposition solution from $1.000 \mathrm{~g}$ of benzoquinone was mixed with $2 \mathrm{ml}$ of $40 \%$ hydrogen peroxide and evaporated slowly on a water-bath. The residue weighed $70 \mathrm{mg}$, but was somewhat contaminated with a yellow peroxidic syrup. A further $0.5 \mathrm{ml}$ of hydrogen peroxide was therefore added together with some water and the mixture again evaporated to dryness. The residue consisted this time of pure, white maleic acid, but weighed only $50 \mathrm{mg}$.

Carbon dioxide. $1.000 \mathrm{~g}$ of benzoquinone was ozonized as usual and the dissolved ozone swept out quantitatively with oxygen. Water was added and the liberated carbon dioxide was carried into a solution of barium hydroxide by the oxygen stream. The precipitated barium carbonate was rapidly filtered off by suction, dried and weighed. Found: $294 \mathrm{mg}$ of barium carbonate which corresponds to $66 \mathrm{mg}$ of carbon dioxide.

Formaldehyde. $260 \mathrm{ml}$ of the chloroform ordinarily used in these experiments were treated with ozone for $6 \mathrm{~h}$ and thereafter extracted five times with $25 \mathrm{ml}$ portions of water. Especially the first extract gave a positive reaction on formaldehyde with chromotropic acid reagent. Further it gave a small crystalline precipitate with an ethanolic solution of dimedone. This product had m. p. $189.5^{\circ}$ which was undepressed upon admixture of an authentic sample.

$500 \mathrm{ml}$ of chloroform were extracted five times with the same volume of water, dried over calcium chloride and distilled. A part of the distillate was treated for $6 \mathrm{~h}$ with ozone and extracted with water. The aqueous extract gave a negative test for formaldehyde. If a small amount of ethanol was added to the purified chloroform and treated with ozone as before, formaldehyde reappeared in the aqueous extracts.

Benzoquinone was dissolved in the purified chloroform and ozonized as usual. The decomposition solution was passed through an ion-exchange column and the neutral eluate collected. With an ethanolic solution of dimedone it gave sparingly a precipitate of white needles. Recrystallized from a small amount of ethanol they had m. p. 190.5 which was undepressed upon admixture of an authentic sample of formaldehyde dimedone derivative. Also the test with chromotropic acid reagent was positive.

\section{REFERENCES}

1. Bernatek, E. Acta Chem. Scand. 8 (1954) 1943.

2. Fichter, Fr., Jetzer, M. and Leepin, R. Ann. 395 (1913) 1.

3. Dallwigk, E. and Briner, E. Helv. Chim. Acta 33 (1950) 2186.

4. Meyer, H. Nachweis und Bestimmung organischer Verbindungen. Lehrbuch der organisch-chemischen Methodik, Julius Springer Verlag, Berlin 1933, Vol. 2, p. 142.

Received June 18, 1958. 